\title{
Covid 19 and its Psychological Resilience among Different Population - A Short Review
}

\author{
Prabhu MR ${ }^{1 *}$, Dwajani $\mathbf{S}^{2}$ and Sahajanand $\mathbf{H}^{3}$ \\ ${ }^{1}$ Research Assistant, Central Research Laboratory, India \\ ${ }^{2}$ Associate Professor, Pharmacology/Senior Research Associate, Central Research \\ Lab, India \\ ${ }^{3}$ Chairperson, Central Research Lab, India \\ *Corresponding Author: Prabhu MR, Research Assistant, Central Research Lab, \\ Rajarajeswari Medical College and Hospital, Karnataka, India.
}

Received: May 12, 2021

Published: June 15, 2021

(C) All rights are reserved by Prabhu MR, et

al.

\begin{abstract}
The COVID-19 pandemic has rapidly spread across the globe causing massive disruptions to everyday life with its impact of health, economics, human behaviors and mental illness. A wide range of mental illness and psychological effects have been observed during COVID-19 pandemic outbreak in all over the global level. The excessive level of stress during COVID-19 pandemic affects healthcare workers and their family members for financial losses contributing to wide spread emotional distress and increase for psychological stress and other mental symptoms. These impacts on healthcare workers may increase their stress and this could be associated with other variables like anxiety and depression.

A significant number of students have also been experiencing psychological distress and mental health issues which were significantly impairing students' academic success, social interactions, future career and personal opportunities. The rapid spread of COVID-19 and social distancing across the country are expected to further affect the mental health of the population including college students. The Elder age groups are more prone to the COVID-19 outbreak due to both clinical and social reasons such as having a weaker immune system, distancing from their families and people with pre-existing respiratory conditions and chronic medical co morbidities. These uncertainty and medical complications, has increased fear about pandemic which has effected on the mental health of senior citizens leading to stress like fear of death, self-negligence, which can in turn lead to non-compliance to the precautions of COVID-19 pandemic. The unaware updates related to the COVID-19 especially for those staying alone have resulted in increase health anxiety and fear.
\end{abstract}

The physical distancing due to COVID-19 outbreak and its lockdown effects can have brought negative impact on the mental health of every individual but majorly in susceptible group of people.

Keywords: Covid 19; Pandemic; Mental Health; Stress; Depression; Anxiety

\section{Introduction}

In December 2019, a new novel Corona virus disease affected and it is believed to have originated from a Wet market in $\mathrm{Wu}$ han, China [1]. The World Health Organization (2020) renamed as a Corona virus disease 2019 (COVID-19) and declared a state of pandemic on $11^{\text {th }}$ March 2020 [2] and spread of this virus became a global threat resulting in large number of hospitalizations and deaths [3]. There are many viruses which were reported in the past 20 years such as influenza, severe acute respiratory syndrome
(SARS), middle east respiratory syndrome (MERS), Ebola, influenza virus with H1N1 Subtypes [4]. Likewise, new novel COVID-19 is a family of virus that can cause from mild illnesses to severe complications such as the common cold to MERS and SARS.

As of April $27^{\text {th }}$, there were millions of confirmed cases with a total of 55,952 deaths in the U.S., according to the Center for Systems Science and Engineering (CSSE) at Johns Hopkins University [5]. In Italy approximately two hundred thousand of people were 
infected due to spread of Corona virus [6]. In India, there were millions of cases confirmed with total of 8313876 and 123611 deaths [7]. The spread of the virus is immensely due to lack of unavailability of medicine and the situation has turned worrisome. Moreover, most of the world's population has been put under lockdown with restricted movement to reduce the rapid spreading of the virus [8] and people have been strictly advised to stay-at-home, closing educational institutions, businesses and public spaces, maintaining social distance, wearing of mask and to sanitize their hands frequently.

In India, entire nation was lockdown from 25 March 2020 to 14 April 2020 and extended later in national response of growing number of cases till 3 May 2020 to reduce the transmission of virus [9]. The signs and symptoms of COVID-19 may appear from 2nd day to 14 days after exposure, called the incubation period. The symptoms can be Fever, Cough, and Tiredness. The early signs and symptoms of Covid-19 may include a loss of taste or smell and other symptoms can include shortness of breath or difficulty breathing, muscles aches, chills, sore throat, runny nose, headache, chest pain and less common symptoms have been reported such as rash, nausea, vomiting, and diarrhea. The severity of COVID-19 symptoms can range from very mild to severe. Older people were more prone to have a higher risk of serious illness from COVID-19 and Children have similar symptoms like adults and generally have mild illness. The person with existing chronic medical conditions such as heart diseases, cancer, Type-2 diabetes, chronic kidney disease, weakened immune system, asthma, liver disease and chronic lung diseases also have a higher risk of serious illness [10].

The COVID-19 pandemic has rapidly spread across the globe causing massive disruptions to everyday life with its impact of health, economics, human behaviors and mental illness [11]. A wide range of mental illness and psychological effects have been observed during COVID-19 pandemic outbreak in all over the global level. At the individual level, people are more scared of hospitalization, getting viral infection or of dying and feeling helpless [12]. The pandemic effect on the public mental health is leading to psychological crises such as anxiety, depression, or insecurity. The widespread fear and high risk perceptions not only leads to mental distress but also drive protective behavior change in response to the threats of medical workers and also in the general population [13]. A study by Brooks., et al. reported the effect of quarantine on the mental health of patient's, with negative impact to psychologi- cal effects including post-traumatic stress symptoms, confusion, anger, mood swing, insomnia, longer quarantine duration, frustration, inadequate information, financial loss [14]. A study by Neir., et al. showed that frequent media exposure may cause distress [15]. There are studies conducted in China which reported that unknown nature of virus can lead to mental disorders due to the pathogenicity of the virus, the rate of virus spread resulting high mortality rate [16].

The COVID-19 pandemic may affect the mental health of individuals at various levels in infected patients, health care workers, families, children, students, patients with mental illness and even workers in other sectors [17]. In this study, we address the effects of COVID-19 pandemic on mental health of different people, its impact on the prevalence of stress, anxiety, and depression, their relationship on psychological resilience of different peoples, behavioral changes and stress since the lockdown.

Effects of pandemic on mental health of health care professionals

Stress classically refers to "bodily process that results from circumstances that plays physical or physiologic demands on an individual". The Previous studies reported with high stress level on health care workers during past pandemic (SARS and MERS) [18]. The excessive level of stress during COVID-19 pandemic affects healthcare workers and their family members for financial losses contributing to wide spread emotional distress and increase for psychological stress and other mental symptoms [19]. These impacts on healthcare workers may increase their stress and this could be associated with other variables like anxiety and depression. A recent study was found that health care workers responsible for the care of COVID-19 patients were more likely to develop symptoms of depression, anxiety, insomnia, and distress [20]. In Indian scenario, many causative factors such as contact with affected patients, forced redeployment to look after affected patients, inadequate training, fear of quarantine particularly in staff with children at home and infected family member and societal stigma against hospital workers seem to be particularly relevant for increased stress level during pandemic [21].

The recent study in India were shown that health care workers working in high-risk hospital based environment demonstrated significantly greater pandemic-related burnout (state of physical, emotional, and mental exhaustion that are emotionally demand- 
ing) compared to personal workers [22]. In particular, health care professionals did not deny in working even in a critical situation, they took risk to be affected by COVID-19; therefore they may be considered as one of the most helpless professionals to develop psychological stress and mental health symptoms like anxiety and depression [23].

\section{Effects of pandemic on mental health of students}

The COVID-19 pandemic affected the school and college students in worldwide by different aspects such as physical, emotional, psychological and financial manner [24]. Moreover, significant number of students was experiencing psychological distress [25] and mental health issues which were significantly impairing students' academic success, social interactions, future career and personal opportunities. The rapid spread of COVID-19 and social distancing across the country are expected to further affect the mental health of the population including college students.

A study conducted in New Jersey have reported that number of students have tested positive for COVID-19 in some schools, colleges and universities [25]. The widespread transmission of the COVID-19 virus, educational institutions across the country have closed and rapidly switched to online learning [26]. In a short period of time, student's lives have dramatically adjusted to new living circumstances and adapt to online learning platforms. The online learning increased stress level among students as they cannot follow the interaction and experiences such as practical labs or artistic performance. Some students may have difficulties with assessed to computer and internet at home $[27,28]$.

In India, the widespread of corona virus transmission has led to school closures and strict containment measures resulting on more families are relying on digital technology to keep children learning, entertained and connected to the world but not all children have the necessary knowledge and skills to keep themselves safe online" [29]. The online learning platforms help the students to get full access of the study material and allow them to engage in online classes and interact with the teachers like the physical classroom setting [30]. The merit of online learning encourages more productive use of time, greater access to experts (nationally and internationally) $24 / 7$ at their own time, teaching various classes simultaneously, useful to some emergency service personnel like police, doctor, nurses and physically handicapped learners who can learn at home. There are several disadvantages on online learning leads to laziness with lack of self discipline, relationship between students and teachers may hamper. Moreover, online classes are explored more hours on computer screen and smart phones due to the pandemic situation resulting headaches, eye problems and stress on their mental health.

A recent study found that more than $50 \%$ of students are affected by stress and their mental health due lockdown and are also stressed for their study because of not comfortable with online classes [31]. In a past study on the stress level of the student in Indian were reported that mild stress was present in $10 \%$, moderate stress was $7.6 \%$ and $6.8 \%$ had severe stress [32]. A study on medical student in Mumbai, India was observed the presence of perceived stress in $73 \%$ of students [33]. A recent paper have examined the stress on the students in accessing campus platforms due to high work load in online learning thus causing a frustration among students and on the other hand system crashes contributing to more stressor [34]. Therefore, the consequences of online learning might affect the student's progress and increased level of stress, anxiety surface and student achievement.

Effects of pandemic on mental health elders and people with disabilities at risk

The Elder age groups are more prone to the COVID-19 outbreak due to both clinical and social reasons such as having a weaker immune system, distancing from their families and people with preexisting respiratory conditions and chronic medical co morbidities. The Elder age group between 60 - 69 years is more likely have COVID-19 infection and may develop serious life threatening condition even if they are in good health and thereby increase fatality rate [35]. There are several factors which have influenced the mortality rate due to COVID-19 such as improper pulmonary involvement, multi organ dysfunction and septicemia in older age group. In recent study, age group of 55 years and above had three times higher risk of mortality and also increased hospitalization, improper pulmonary involvement, delayed recovery, acute disease progression and increased comorbidities of diabetes, hypertension and cardiac problems [36]. These uncertainty and medical complications has increased fear about pandemic which has effected on the mental health of senior citizens leading to stress like fear of death, selfnegligence, which can in turn lead to non-compliance to the precautions of COVID-19 pandemic. The unaware updates related to the COVID-19 especially for those staying alone have resulted in increase health anxiety and fear. 
On the other hand, the effects of quarantine leading to loneliness, social distancing from their families and friends can have long-lasting psychological effects and loneliness is a potent risk factor for anxiety, depression and cognitive disorders with lack of physical activity [37]. A recent study showed increased incidence of depressive disorder and posttraumatic stress disorder in the elder groups [38]. People those who are physically challenged may have an impact of causing mental illness during COVID-19 crisis. A study showed that disabled people harder to access critical medical supplies which can become even more challenging in past pandemics [39]. There are some people with disabilities which were reported with higher levels of social isolation resulting intensified feelings of loneliness and physical distancing have been associated with increases in stress, anxiety, heart disease, dementia and other health problems [40]. The adequate research on the impact of COVID-19 on disability community is not yet available due to lack of data on rates of infections, outcomes and deaths disaggregated by disability, age, race and income and among other factors.

However, many people with disabilities are healthy while some have underlying secondary aspect of their disability such as suppressed immune systems or respiratory problems, skin breakdown or urinary tract infection have an increase risk of severe symptoms associated with COVID-19 [20]. The current pandemic can cause additional stress and fears of people with disability. A recent study conducted in Nepal, India was reported that elevated levels of anxiety and depression than stress was reported by the participants with different levels of disability. Significantly, the highest levels of depression, anxiety, and stress were reported by participants with mental or psychosocial disabilities and least level of anxiety, depression was reported by participants with intellectual disability. The highest level of perceived social support was reported to voice and speech related disability group and the lowest with intellectual disability [41].

\section{Psychological resilience in the COVID-19 pandemic}

Psychologists defined resilience as the process of adapting well in the face of adversity, trauma, tragedy, threats or significant sources of stress - such as family and relationship problems, serious health problems or work place and financial stresses. As much as resilience involved "bouncing back" from these difficult experiences it can also involve profound personal growth. The healthcare workers are facing tremendous pressure from COVID-19, especially those who are in contact with suspected or confirmed cases due to high risk of infection inadequate protection, lack of experience in managing the diseases, significant life style changes [20]. These factors increased the incidence of psychological problems among health care workers such as fear, anxiety, depression and insomnia which can negatively affects work efficiency and long-term wellbeing [42].

\section{Conclusion}

Children are likely to develop worry, anxiety and fear; this can include the types of fears that are experienced by adults such as $\mathrm{S}$ fear of dying, fear of their relatives dying or a fear of what it means to receive medical treatments. If schools have closed, then children may no longer have fear provided by that environment, now they have less opportunity to be with their friends and get that social support that is essential for good mental well-being. The physical distancing due to COVID-19 outbreak can have negative impact on the mental health of the elderly and disabled individual. Physical isolation at home among family members can put a high risk on mental health. It can cause anxiety and stress and traumatic situation for them. Elderly people depend on young ones for their daily need and self isolation can critically damage the family system. The family members may witness irritating and shouting behavior.

\section{Bibliography}

1. Bai Y., et al. "Survey of stress reactions among health care workers involved with the SARS outbreak". Psychiatric Services 55.9 (2004): 1055-1057.

2. WHO. WHO Coronavirus Disease (COVID-19) Dashboard; WHO: Geneva, Switzerland (2020).

3. Wang C., et al. "A novel coronavirus outbreak of global health concern". Lancet 395.10223 (2020): 470-473.

4. Ashour HM., et al. "Insights into the recent 2019 novel coronavirus (SARS-CoV-2) in light of past human coronavirus outbreaks". Pathogens 9.3 (2020): 186.

5. New Jersey Department of Health. "New Jersey COVID-19 Information Hub" (2020).

6. Alessandra Babore., et al. "Psychological effects of the COVID-2019 pandemic: Perceived stress and coping strategies among healthcare professionals". Psychiatry Research 293 (2020): 113366.

7. Number of COVID-19 cases in india climbs to 467, Death toll rises to nine. Livemint (2020). 
8. Kaplan J., et al. "A third of the global population is on coronavirus lockdown - Here's our constantly updated list of countries and restrictions" (2020).

9. Shankar Das. "Mental Health and Psychosocial Aspects of COVID-19 in India: The Challenges and Responses". Journal of Health Management 22.2 (2020): 197-205.

10. Chen N., et al. "Epidemiological and clinical characteristics of 99 cases of 2019 novel coronavirus pneumonia in Wuhan, China: a descriptive study". Lancet 395.10223 (2020): 507-513.

11. Holmes EA., et al. "Multidisciplinary research priorities for the COVID-19 pandemic: A call for action for mental health science". Lancet Psychiatry 7 (2020): 547-560.

12. Hall RC., et al. "The 1995 Kikwit Ebola outbreak: lessons hospitals and physicians can apply to future viral epidemics". General Hospital Psychiatry 30.5 (2008): 446-452.

13. Bavel JJV., et al. "Using social and behavioural science to support COVID-19 pandemic response". Nature Human Behaviour 4 (2020): 460-471.

14. Brooks S K., et al. "The psychological impact of quarantine and how to reduce it: Rapid review of the evidence". Lancet 395 (2020): 912-920.

15. Neria Y and Sullivan GM. "Understanding the mental health effects of indirect exposure to mass trauma through the media". JAMA 306.12 (2011): 1374-1375.

16. Shigemura J., et al. "Public responses to the novel 2019 coronavirus (2019-nCoV) in Japan: mental health consequences and target populations". Psychiatry and Clinical Neurosciences 74.4 (2020): 281.

17. Ryu S., et al. "An interim review of the epidemiological characteristics of 2019 novel coronavirus". Epidemiology Health 42 (2020): e2020006.

18. Healy S., et al. "Stress in emergency departments: experiences of nurses and doctors". Emergency Nurse 19.4 (2011): 31-37.

19. Verma S., et al. "Post- SARS psychological morbidity and stigma among general practitioners and traditional Chinese medicine practitioners in Singapore". Annals of the Academy of Medicine, Singapore 33.6 (2004): 743-748.

20. Lai J., et al. "Factors associated with mental health outcomes among health care workers exposed to coronavirus disease 2019". JAMA Network Open 3.3 (2020): e203976.
21. Kisely S., et al. "Occurrence, prevention, and management of the psychological effects of emerging virus outbreaks on healthcare workers: rapid review and meta-analysis". BMJ 369 (2020): m1642.

22. Ruchira W Khasne., et al. "Burnout among Healthcare Workers during COVID-19 Pandemic in India: Results of a Questionnaire-based Survey". Indian Journal of Critical Care Medicine 24.8 (2020): 664-671.

23. Khalid I., et al. "Healthcare workers emotions, perceived stressors and coping strategies during a MERS-CoV outbreak". Clinical Medicine and Research. 14.1 (2016): 7-14.

24. Aleksandar Kecojevic., et al. "The impact of the COVID-19 epidemic on mental health of undergraduate students in New Jersey, cross-sectional study". PLoS ONE 15.9 (2020): e0239696.

25. American College Health Association. American College Health Association-National College Health Assessment III: Reference Group Executive Summary Fall 2019. Silver Spring, MD (2020).

26. Bloomfield College. Important Update On COVID-19 At Bloomfield College, Student Tests Positive TAP into (2020). Available from: important-update-on-covid-19-at-bloomfield-collegestudent-tests-positive

27. Gewin V. "Five tips for moving teaching online as COVID-19 takes hold”. Nature 580.7802 (2020): 295-296.

28. Dwajani S., et al. "Effect of increased screen time in undergraduate students during covid-19 pandemic-a survey-based study". International Journal of Recent Scientific Research 11.12B (2020): 40252-40258.

29. Lederman D. "How the shift to remote learning might affect students, instructors and colleges" (2020).

30. "UGC forms committee to encourage online learning amid lockdown" (2020).

31. Ambreen Fatima and Utsav raj. "Stress in students after lockdown due to COVID-19 thereat and The effects of attending online classes". SSRN Electronic Journal (2020).

32. Vivek B Waghachavare., et al. "A Study of Stress among Students of Professional Colleges from an Urban area in India". Sultan Qaboos University Medical Journal 13.3 (2013): 429436. 
33. Supe AN. "A study of stress in medical students at Seth G.S. Medical College". Journal of Postgraduate Medicine 44 (1998): 1-6.

34. Mirna Fawaz Ali Samaha. "E-learning: Depression, anxiety, Stress related symptomatology among Lebanese university students during COVID-19 Pandemic". Nursing Forum (2020): 1-6.

35. Wu Z and McGoogan JM. "Characteristics of and important lessons from the coronavirus disease 2019 (COVID-19) outbreak in China: summary of a report of 72314 cases from the Chinese Center for Disease Control and Prevention". JAMA 323.13 (2020): 1239-1242.

36. Liu K., et al. "Clinical features of COVID-19 in elderly patients: a comparison with young and middle-aged patients". Journal of Infection 80.6 (2020): 14-18.

37. Aylaz R., et al. "Relationship between depression and loneliness in elderly and examination of influential factors". Archives of Gerontology and Geriatrics 55.3 (2012): 548-554.

38. Banerjee D. "How COVID-19 is overwhelming our mental health". Nature India (2020).

39. Campbell V A., et al. "Preparing for and responding to pandemic influenza: Implications for people with disabilities”. American Journal of Public Health 99 (2009): S294-S300.

40. National Academies of Science, Engineering, and Medicine. Social isolation and loneliness in older adults: Opportunities for the health care system. Washington, DC: National Academies Press (2020).

41. Sandesh Dhakal., et al. "Mental health and psychosocial impact of COVID-19 and lockdown on persons with disabilities in Nepal". National Mental Health Self Help Organization In collaboration with National Federation of the Disabled Nepal and CBM (2020).

42. Pappa S., et al. "Prevalence of depression, anxiety, and insomnia among healthcare workers during the COVID-19 pandemic: a systematic review and metaanalysis". Brain, Behavior, and Immunity 88 (2020): 901-907.

Volume 4 Issue 7 July 2021

(C) All rights are reserved by Prabhu MR., et al. 